\title{
A MANDELBROT SET WHOSE BOUNDARY IS PIECEWISE SMOOTH
}

\author{
M. F. BARNSLEY AND D. P. HARDIN
}

\begin{abstract}
It is proved that the Mandelbrot set associated with the pair of maps $w_{1,2}: \mathbf{C} \rightarrow \mathbf{C}, w_{1}(z)=s z+1, w_{2}(z)=s^{*} z-1$, with parameter $s \in \mathbf{C}$, is connected and has piecewise smooth boundary.
\end{abstract}

\section{INTRODUCTION}

The discovery [1] of the Mandelbrot set $M$ for the iterated complex polynomial $z^{2}+s$ has generated considerable research activity $[2,3]$, especially because of its relation to cascades of bifurcations and universal phenomena [4].

The Mandelbrot set $M$ consists of those values of $s \in \mathbf{C}$ such that the Julia set $J(s)$ for $z^{2}-s$ is connected. Barnsley and Harrington [5] considered an analogous Mandelbrot set $D$ associated with the two affine maps $T_{1,2}: \mathrm{C} \rightarrow \mathrm{C}$ defined by

$$
T_{1}(z)=s z+1, \quad T_{2}(z)=s z-1
$$

for $s \in \mathbf{C}$ and $|s|<1$. There is a unique nonempty compact set $A(s)$ which is invariant under $T_{1}$ and $T_{2}$ (i.e., $T_{1}(A(s)) \cup T_{2}(A(s)=A(s)$ ) [5,6]. Generically, $A(s)$ is a fractal. $D$ is defined to be the set of $s \in \mathbf{C},|s|<1$ for which $A(s)$ is disconnected. The boundary of $D$ contains self-similar structures (see Figure 2) and appears to be a fractal. It is not known whether $D$ is connected; however, new pictures of this set presented here indicate that it is.

In this paper we study the Mandelbrot set $G$ associated with the two affine maps $w_{1,2}: \mathbf{C} \rightarrow \mathbf{C}$ defined by

$$
w_{1}(z)=s z+1, \quad w_{2}(z)=s^{*} z-1
$$

for $s \in \mathbf{C}$ with $|s|<1$. (Here $s^{*}$ denotes the conjugate of $s$.) As in the previous case, there is a unique invariant compact set $A(s)$ which is generically a fractal. Despite the apparent similarity between the two pairs of maps, $G$ is easier to analyze than $D$. We will show among other things that $G$ is connected and, remarkably, has a piecewise smooth boundary. Pictures of the associated fractals as one travels around the boundary of $G$ are given.

Received by the editors November 23, 1987.

1980 Mathematics Subject Classification (1985 Revision). Primary 40A99, 51 M99.

Research by M. F. Barnsley supported in part by DARPA "Image Compression Project," ONR contract N00014-86-C-0446. 


\section{Preliminaries}

Let $(X, d)$ be a compact metric space or $\mathbf{R}^{n}$ and let $H$ denote the set of all nonempty compact subsets of $X$. If $B, C \subset X$, then define

$$
d(B, C)=\inf \{d(b, c) \mid b \in B, c \in C\}
$$

and define the Hausdorff metric $h$ on $H$ by

$$
h(B, C)=\sup \{d(\{b\}, C), d(\{c\}, B) \mid b \in B, c \in C\} .
$$

It is known that $(H, h)$ is a complete metric space [6].

Let $0 \leq c<1$ and let the mappings $w_{i}: X \rightarrow X, i=1, \ldots, N$, be such that $d\left(w_{i}(x), w_{i}(y)\right) \leq c d(x, y)$ for all $x, y \in X$. Following Barnsley and Demko [7] we call $\left\{X, w_{i}: i=1, \ldots, N\right\}$ a hyperbolic iterated function system (HIFS). Define $\underline{w}: H \rightarrow H$ by

$$
\underline{w}(B)=\bigcup_{i=1}^{N} w_{i}(B)=\bigcup\left\{w_{i}(x) \mid x \in B, i \in[1, \ldots, N]\right\}
$$

for all $B \in H$. From the definition of $h$ it is immediate that $\underline{w}$ is a contraction on $H$ with $h\left(\underline{w}\left(B_{1}\right), \underline{w}\left(B_{2}\right)\right) \leq \operatorname{ch}\left(B_{1}, B_{2}\right)$ for $B_{1}, B_{2} \in H$. Since $H$ is complete, the Banach fixed point theorem implies

Theorem 1. (1) $\underline{w}$ has a unique fixed point $A \in H$. ( $A$ is called the attractor for the HIFS $(X, \underline{w})$.)

(2) $\lim _{n \rightarrow \infty} \underline{w}^{\circ n}(B)=A$ (i.e., $\lim _{n \rightarrow \infty} h\left(\underline{w}^{\circ n}(B), A\right)=0$ ) for any $B \in H$, where we define $\underline{w}^{\circ 0}(B)=B$ and $\underline{w}^{\circ n}(B)=\underline{w}\left(\underline{w}^{\circ(n-1)}(B)\right)$ for $n \in \mathbf{N}$.

We will need the following lemma.

Lemma 2. If $B \in H$ and $B \supset \underline{w}(B)$ then $\underline{w}^{\circ n}(B) \supset A$ for all $n \in \mathbf{N}$, where $A$ is the attractor for $\{X, \underline{w}\}$. If $\underline{w}^{\circ n}(B)$ is connected for all $n \in \mathbf{N}$ and some $B \subset H$ then $A$ is connected.

Proof. If $\underline{w}(B) \subset B$, then $\underline{w}^{\circ n}(B) \subset \underline{w}^{\circ(n-1)}(B)$ for all $n \in \mathbf{N}$. Thus $A=$ $\lim _{n \rightarrow \infty} \underline{w}^{\circ n}(B)=\bigcap_{n=1}^{\infty} \underline{w}^{\circ n}(B)$, because the sequence of compact sets $\left\{\underline{w}^{\circ n}(B)\right\}$ is decreasing.

Suppose $A$ is disconnected; then $A=B_{1} \cup B_{2}$ with $B_{1}, B_{2} \in H$ and $B_{1} \cap B_{2}=\varnothing$. Thus $d\left(B_{1}, B_{2}\right)>0$ and so for any set $C$ such that $h(A, C)<$ $d\left(B_{1}, B_{2}\right) / 2$ then $C$ is also disconnected. Since $\lim _{n \rightarrow \infty} h\left(A, \underline{w}^{\circ n}(B)\right)=0$, $\underline{w}^{\circ n}(B)$ is eventually disconnected for any $B \in H$.

The following corollary generalizes a result of Barnsley and Harrington [5].

Corollary 3. Let $\left(X, w_{1}, w_{2}\right)$ be an HIFS with attractor $A$ such that there exists a nonempty connected $B \in H$ with $\underline{w}(B) \subset B . A$ is disconnected if and only if $w_{1}(A) \cap w_{2}(A)=\varnothing$.

Proof. If $w_{1}(A) \cap w_{2}(A)=\varnothing$ then $w_{1}(A)$ and $w_{2}(A)$ form a disconnection of A. 
Suppose $w_{1}(A) \cap w_{2}(A) \neq \varnothing$. By Lemma $2, \underline{w}^{\circ n}(B) \supset A$. Suppose $\underline{w}^{\circ n}(B)$ is connected; then $w_{1}\left(\underline{w}^{\circ n}(B)\right) \cap w_{2}\left(\underline{w}^{o n}(B)\right) \supset w_{1}(A) \cap w_{2}(A) \neq \varnothing$. By continuity $w_{1}\left(\underline{w}^{o n}(B)\right)$ and $w_{2}\left(\underline{w}^{\circ n}(B)\right)$ are connected so $\underline{w}^{o(n+1)}(B)$ is connected. Since $B$ is connected, $\underline{w}^{o(n)}(B)$ is connected for all $n \in \mathbf{N}$ by induction. By Theorem 1, $\lim _{n \rightarrow \infty} h\left(\underline{w}^{o n}(B), A\right)=0$ and so by Lemma $2, A$ is connected.

Note that if $X=\mathbf{R}^{n}$ then we can always find a nonempty connected $B \in H$ such that $\underline{w}(B) \subset B$; for instance, if we pick the radius large enough we can take $B$ to be a closed ball centered at the origin.

If $(X, \underline{w}(\lambda, \cdot))$ is an HIFS for each $\lambda$ in an index set $\Lambda$, then we define the Mandelbrot set for the family $\{(X, \underline{w}(\lambda, \cdot)) \mid \lambda \in \Lambda\}$ to be the set of $\lambda \in \Lambda$ for which $A(\lambda)$ (i.e., the attractor for $(X, \underline{w}(\lambda, \cdot)))$ is disconnected.

\section{A PREVIOUSLY CONSIDERED MANDELBROT SET}

Consider the family of pairs of maps $T_{i}: \mathbf{C} \rightarrow \mathbf{C}, i=1,2$, defined by

$$
T_{1}(s, \cdot): z \rightarrow s z+1, \quad T_{2}(s, \cdot): z \rightarrow s z-1
$$

for $s \in \mathbf{C}$ and $|s|<1$. Note that $T_{1}$ and $T_{2}$ are similitudes. Let $\theta(s)=\arg (s)$ and suppose $B \subset K$. Geometrically, $\underline{T}(s, B)=T_{1}(s, B) \cup T_{2}(s, B)$ is generated by shrinking $B$ by $|s|$ toward 0 , rotating by $\theta(s)$ about 0 , and translating one such copy by $1+i 0$ and another by $-1+i 0$.

Since $\left|T_{i}(s, a)-T_{i}(s, b)\right|=|s||a-b|$ for $i=1,2$ and $a, b \in \mathbf{C}$, we see that $\left(\mathrm{C}, T_{1}(s, \cdot), T_{2}(s, \cdot)\right)$ is an HIFS for $|s|<1$. Let $A(s)$ denote the attractor for this HIFS. Figure 1 shows $A(s)$ for several values of $s$. It is instructive to identify $T_{1}(s, A(s))$ and $T_{2}(s, A(s))$ and to note that $A(s)$ is indeed the fixed point of $\underline{T}(s, \cdot)$.

Barnsley and Demko [7] investigated the Mandelbrot set for the family of HIFSs $\{(\mathbf{C}, \underline{T}(s, \cdot))|s \in \mathbf{C}| s \mid,<1\}$. We will denote this Mandelbrot set by $D$. Figure 2 shows a computer-generated picture of $D$ (from [7]) along with several blowups of portions of the boundary of $D$. They hypothesized that $D$ may be disconnected; however, Figure 2 suggests that the opposite may be true.

They found inner and outer bounds for $D$ using the fact that if $s \in D$ then the Hausdorff dimension $d$ of $A(s)$ is given by

$$
d=\log \left(\frac{1}{2}\right) / \log (|s|) \text {. }
$$

We will prove the same bounds for $D$ using the results we developed in the previous section. In the following, we will suppress the $s$ dependence of $T_{1}$ and $T_{2}$.

Proposition 4. If $|s|<.5$ then $s \in D$.

Proof. Let $R_{s}=1 /(1-|s|)$ and $B(x, r)=\{z \in \mathbf{C}|| z-x \mid \leq r\}$. Then $T_{1}\left(B\left(0, R_{s}\right)\right)=B\left(1,|s| R_{s}\right) \subset B\left(0, R_{s}\right)$ and $T_{2}\left(B\left(0, R_{s}\right)\right)=B\left(-1,|s| R_{s}\right)$ (see Figure 3). Thus, $\underline{T}\left(B\left(0, R_{s}\right)\right) \subset B\left(0, R_{s}\right)$ and, by Lemma $2, A(s) \subset B\left(0, R_{s}\right)$. If $|s|<.5$ then $|s| R_{s}<1$ and so $B\left(-1,|s| R_{s}\right) \cap B\left(1,|s| R_{s}\right)=\varnothing$. Thus $T_{1}(A(s)) \cap T_{2}(A(s))=\varnothing$ and $A(s)$ is disconnected. 
Re: : 7399865
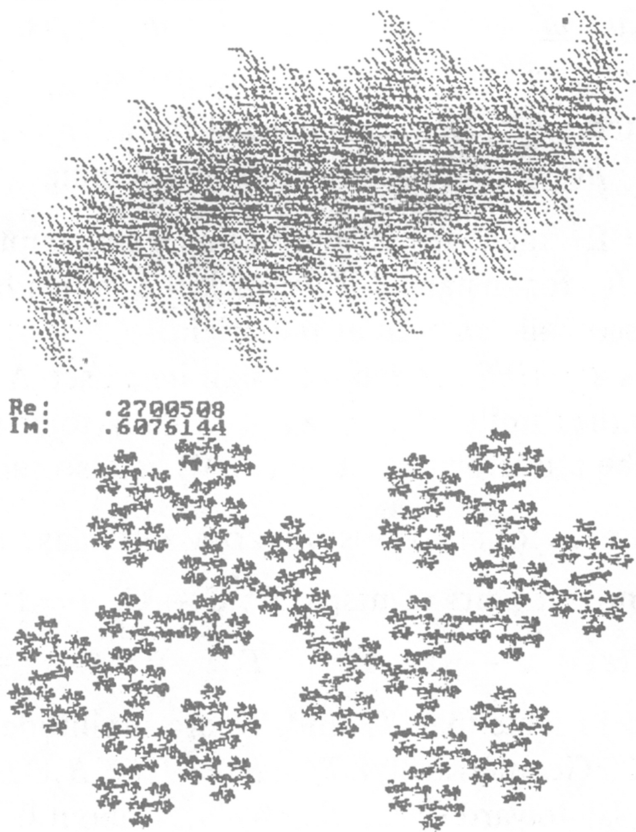

Re: $: 4974621$

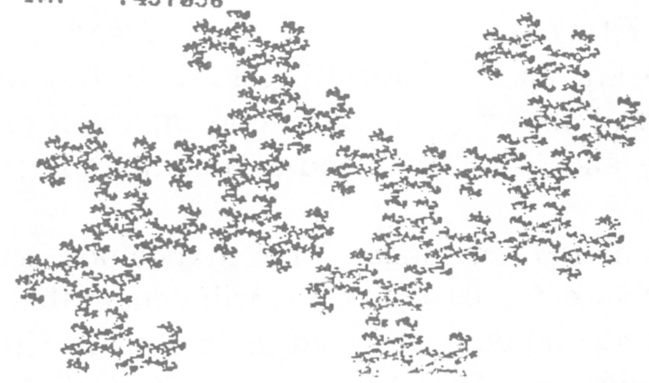

FIGURE 1. The attractor $A(s)$ for $I$ shown for various values of $s$

We can calculate successively better inner bounds for $D$ by determining the values of $s$ for which it is true that $T_{1}\left(\underline{T}^{\circ n}\left(B\left(0, R_{s}\right)\right)\right) \cap T_{2}\left(\underline{T}^{\circ n}\left(B\left(0, R_{s}\right)\right)\right)=\varnothing$ for successively larger values of $n$. In fact, all of $D$ can be calculated in this manner.

Theorem 5. $s \in D$ if and only if $T_{1}\left(\underline{T}^{\circ n}\left(B\left(0, R_{s}\right)\right)\right) \cap T_{2}\left(\underline{T}^{\circ n}\left(B\left(0, R_{s}\right)\right)\right)=\varnothing$ for some $n \in \mathbf{N}$.

Proof. Let $B=B\left(0, R_{s}\right)$ and $B_{n}=\underline{T}^{\circ n}\left(B\left(0, R_{s}\right)\right)$ for $n \in \mathbf{N}$.

By Lemma 2, $A(s) \subset B_{n}$ for all $n \in \mathbf{N}$, so $A(s)$ is disconnected if $T_{1}\left(B_{n}\right) \cap$ $T_{2}\left(B_{n}\right)=\varnothing$ for some $n \in \mathbf{N}$.

If $T_{1}\left(B_{n}\right) \cap T_{2}\left(B_{n}\right) \neq \varnothing$ for all $n \in \mathbf{N}$, then since $T_{1}$ and $T_{2}$ are continuous and $B$ is connected, we get, via an induction, that $B_{n}$ is connected. By Lemma 2, $A(s)$ is connected. 


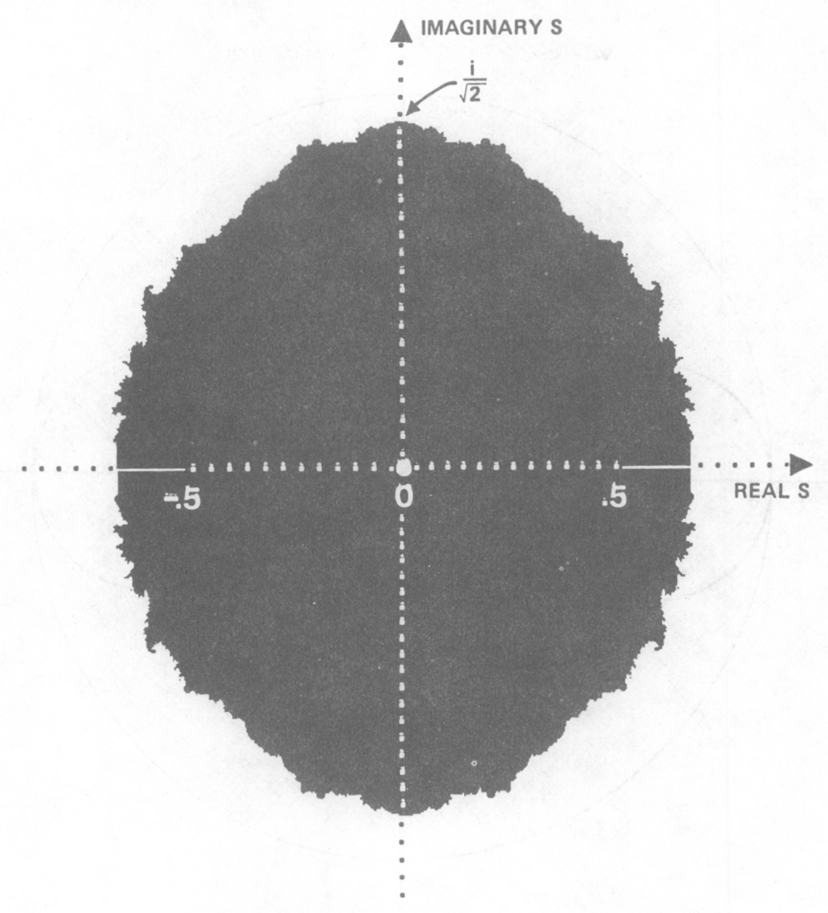

(a) The Mandelbrot set $D$

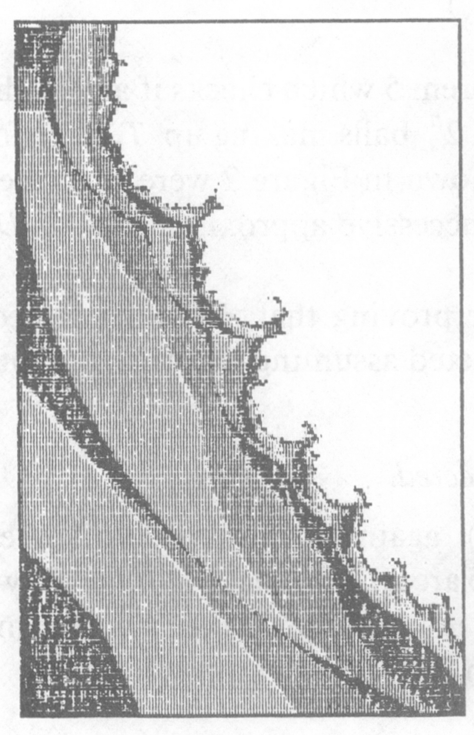

(b) Blowup of part of $D$, where $.49 \leq \operatorname{Re}[s] \leq .55$ and $.35 \leq \operatorname{Im}[s] \leq .45$

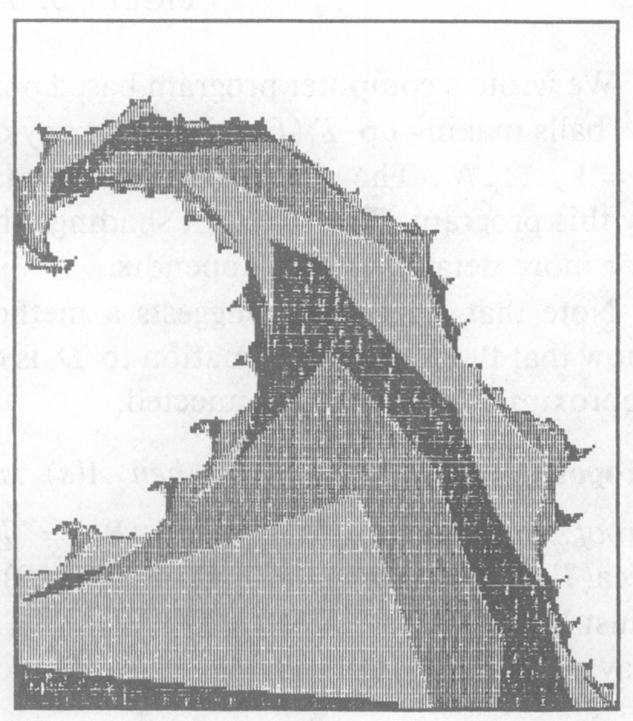

(c) Blowup of part of $D$, where $.572 \leq \operatorname{Re}[s] \leq .593$ and $.352 \leq \operatorname{Im}[s] \leq .378$ 


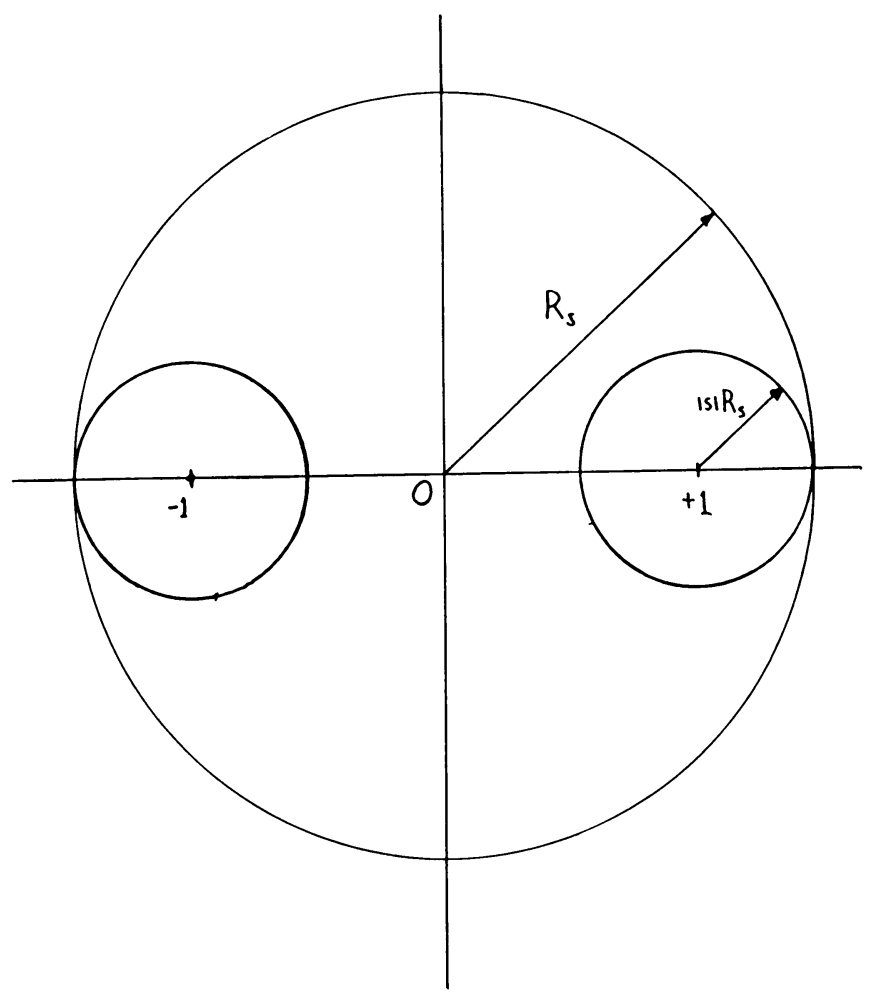

FiguRE 3. $B\left(0, R_{s}\right)$

We wrote a computer program based on Theorem 5 which checks if any of the $2^{n}$ balls making up $T_{1}\left(B_{n}\right)$ intersect any of the $2^{n}$ balls making up $T_{2}\left(B_{n}\right)$ for $n=1, \ldots, N$. The pictures of the boundary shown in Figure 2 were generated by this program. The different shadings show successive approximations to $D$. For more details, see the appendix.

Note that Theorem 5 suggests a method for proving that $D$ is connected: show that the $n$th approximation to $D$ is connected assuming that the $(n-1)$ th approximation to $D$ is connected.

Proposition 6. If $|s|<1 / \sqrt{2}$ then $A(s)$ is connected.

Proof. Let $B=B\left(0, R_{s}\right)$ and $B_{n}=\underline{T}^{\circ n}(B)$ again. If $|s|>1 / \sqrt{2}$ then $\operatorname{area}\left(T_{1}(B)\right)>.5 \operatorname{area}(B)$ and $\operatorname{area}\left(T_{2}(B)\right)>.5 \operatorname{area}(B)$. Since $\underline{T}(B) \subset B$ we must have $T_{1}(B) \cap T_{2}(B) \neq \varnothing$. Since $\underline{T}\left(B_{n}\right) \subset B_{n}$ we must have, in the same way, that $T_{1}\left(B_{n}\right) \cap T_{2}\left(B_{n}\right) \neq \varnothing$. By Proposition 5, $A(s)$ is connected.

Proposition 6 can be generalized to get

Proposition 7. If $\left(\mathbf{R}^{n}, w_{1}, w_{2}\right)$ is an HIFS such that $\operatorname{vol}_{n}\left(w_{1}(B)\right)>\frac{1}{2} \operatorname{vol}_{n}(B)$ for every set $B \in \mathbf{R}^{n}$ with finite and nonzero n-dimensional volume $\operatorname{vol}_{n}(B)$, then the attractor $A$ is connected. 
3. A PIECEWISE SMOOTh MANDELbRot SET

Now consider the family of HIFSs arising from the pair of maps $w_{i}: \mathbf{C} \rightarrow \mathbf{C}$, $i=1,2$, defined by

$$
w_{1}(s, \cdot): z \rightarrow s z+1, \quad w_{2}(s, \cdot): z \rightarrow s^{*} z-1
$$

for $s \in \mathbf{C}$ and $|s|<1$.

\section{Re $[S]=: 4900001$}

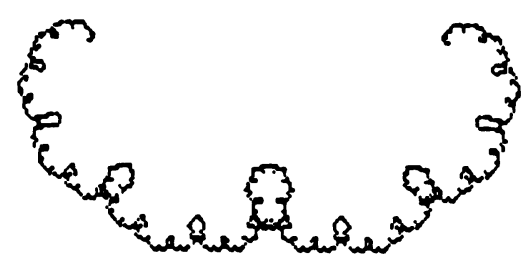

$\operatorname{Re}[S]=: \frac{17}{5778999}$

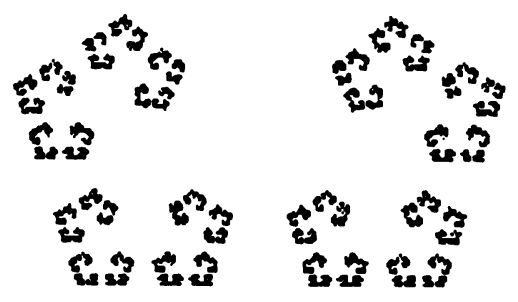

$\operatorname{Re}[\$]=-: 227$

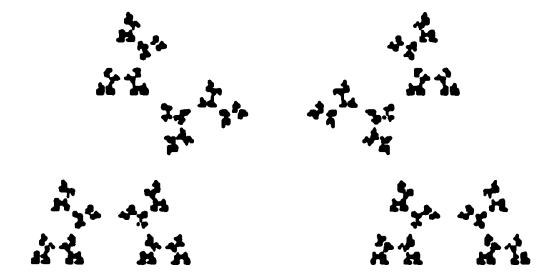

FIGURE 4. $A(s)$ for various values of $s$ 


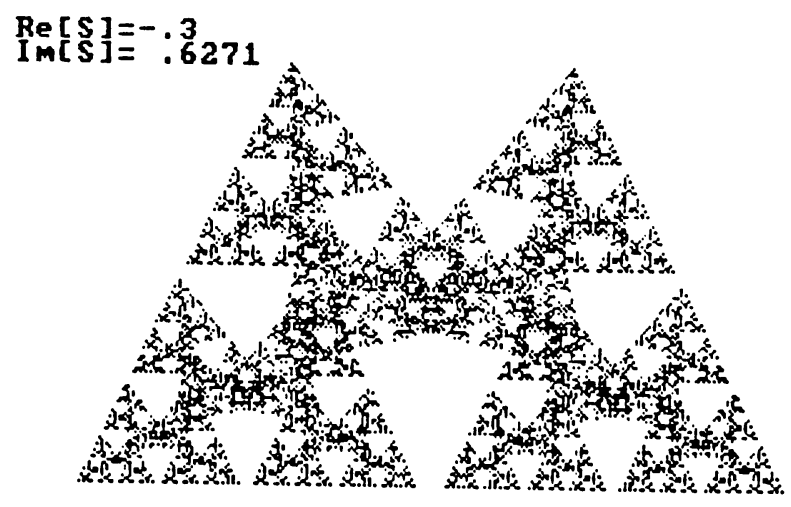

\section{$\operatorname{Re}[S]=-.4424$}

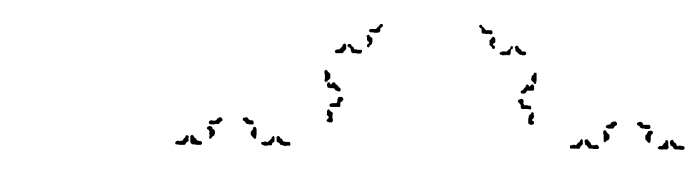

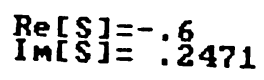

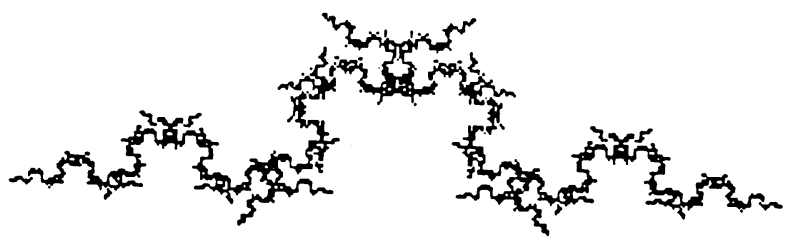

FIGURE 4 (continued)

Geometrically, $\underline{w}$ acts on a set $B$ in almost the same way as $\underline{T}$, the difference being that one of the shrunken copies is rotated by $-\theta(s)$ where $\theta(s)=$ $\arg (s)$ again. Figure 4 shows $A(s)$ for various values of $s$.

Let $G$ denote the Mandelbrot set for $\{(\mathbf{C}, \underline{w}(s, \cdot))|s \in \mathbf{C}| s \mid,<1\}$. As we shall see, in contrast to all other known cases, $G$ can be completely described in an elementary way. We will show that $G$ is connected and that the boundary of $G$ is a countable collection of pieces of polynomial curves in $x=\operatorname{Re}[s]$ and $y=\operatorname{Im}[s]$. Figure 5 shows a picture of $G$. Note that the inner and outer bounds for $D$ are also applicable to $G$ by exactly the same arguments.

First we will prove that $G$ is symmetric about the real axis. 


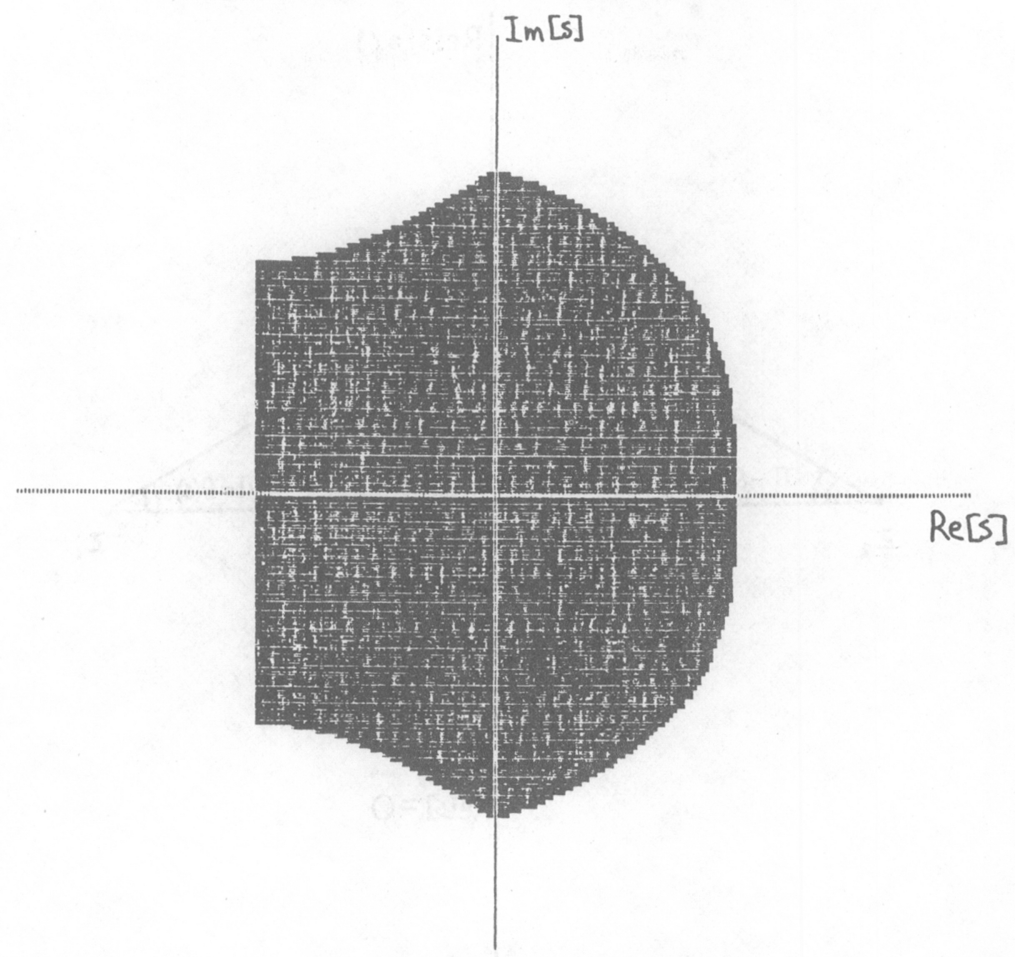

FIGURE 5. The Mandelbrot set $G$

Proposition 8. $A\left(s^{*}\right)=-A(s)$ so $s \in G$ if and only if $s^{*} \in G$.

Proof. $A(s)$ satisfies $\underline{w}(s, A(s))=(s A(s)+1) \cup\left(s^{*} A(s)-1\right)=A(s)$. Thus, $-A(s)=(s(-A(s))-1) \cup\left(s^{*}(-A(s))+1\right)=w_{2}\left(s^{*},-A(s)\right) \cup w_{1}\left(s^{*},-A(s)\right)=$ $\underline{w}\left(s^{*},-A(s)\right)$. Since $\underline{w}\left(s^{*}, \cdot\right)$ has a unique fixed point, $-A(s)=A\left(s^{*}\right)$.

Let $z_{1}$ be the fixed point of the contraction $w_{1} \circ w_{2}$ and $z_{2}$ be the fixed point of $w_{2} \circ w_{1}$. We will need the following collection of facts, which follow directly from the definitions of $w_{1}$ and $w_{2}$. Hereafter we will suppress the $s$ dependence of $w_{1}$ and $w_{2}$.

\section{Lemma 9.}

(a) $z_{1}=(1-s) /\left(1-|s|^{2}\right)=-z_{2}^{*}=w_{1}\left(z_{2}\right)$.

(b) $z_{2}=\left(s^{*}-1\right) /\left(1-|s|^{2}\right)=-z_{1}^{*}=w_{2}\left(z_{1}\right)$.

(c) $-w_{1}(x)^{*}=w_{2}\left(-z^{*}\right)$ for $z \in \mathbf{C}$.

(d) From the above we get

(i) $w_{2}\left(z_{2}\right)=-w_{1}\left(z_{1}\right)^{*}$ and

(ii) $w_{2} \circ w_{2}\left(z_{2}\right)=-\left(w_{1} \circ w_{1}\left(z_{1}\right)\right)^{*}$.

Proposition 10. If $3 \pi / 4 \leq \theta(s) \leq \pi$ and $\operatorname{Re}\left[w_{1}\left(z_{1}\right)\right]>0$ then $A(s)$ is disconnected.

Proof. Since $|s|<1$, it is clear that $\operatorname{Re}\left[z_{1}\right]=\operatorname{Re}\left[(1-s) /\left(1-|s|^{2}\right)\right]>0$. Let $B$ denote the closed convex hull of $\left\{z_{1}, z_{2}, w_{1}\left(z_{1}\right), w_{2}\left(z_{2}\right)\right\}$. The idea of 


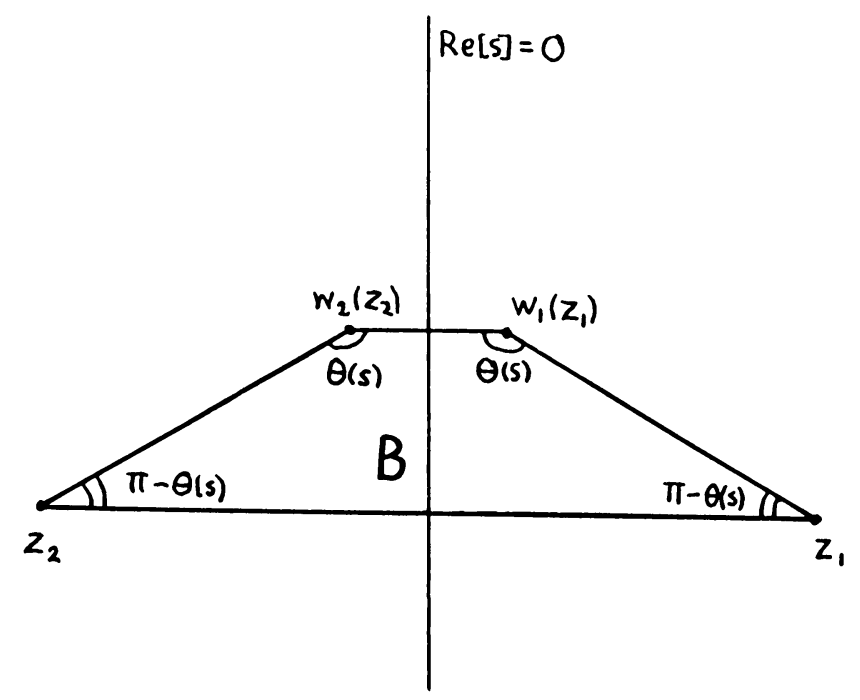

FIGURE 6

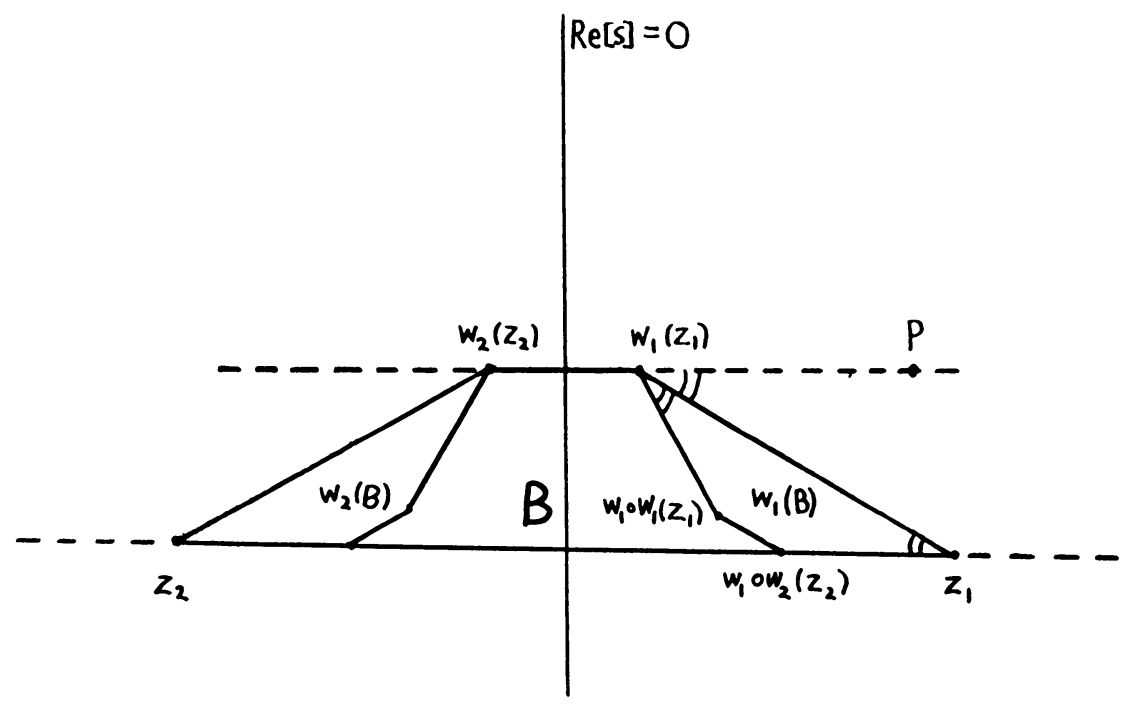

FIGURE 7

the proof is to show that $\underline{w}(B) \subset B$ so that $A \subset B$ and then to show that $w_{1}(B) \cap w_{2}(B)=\varnothing$.

Lemma 9 gave $z_{2}=-z_{1}^{*}$ and $w_{2}\left(z_{2}\right)=-\left(w_{1}\left(z_{1}\right)\right)^{*}$ so $B$ is a trapezoid as shown in Figure 6. Since $w_{1}\left(\overline{z_{2} z_{1}}\right)=\overline{z_{1} w_{1}\left(z_{1}\right)}$, then from the definition of $w_{1}$ we see that the vertex angle at $w_{1}\left(z_{1}\right)$ is $\theta(s)$ and the vertex angle at $z_{1}$ is $\pi-\theta(s)$. By symmetry the vertex angle at $z_{2}$ is $\pi-\theta(s)$ and the vertex angle at $w_{2}\left(z_{2}\right)$ is $\theta(s)$.

Consider Figure 7. Since the angle $/ z_{1} z_{2} w_{2}\left(z_{2}\right)$ measured from $\overline{z_{2} z_{1}}$ in a counterclockwise direction is $\pi-\theta(s)$ (i.e., the vertex angle at $z_{2}$ ) we see that 

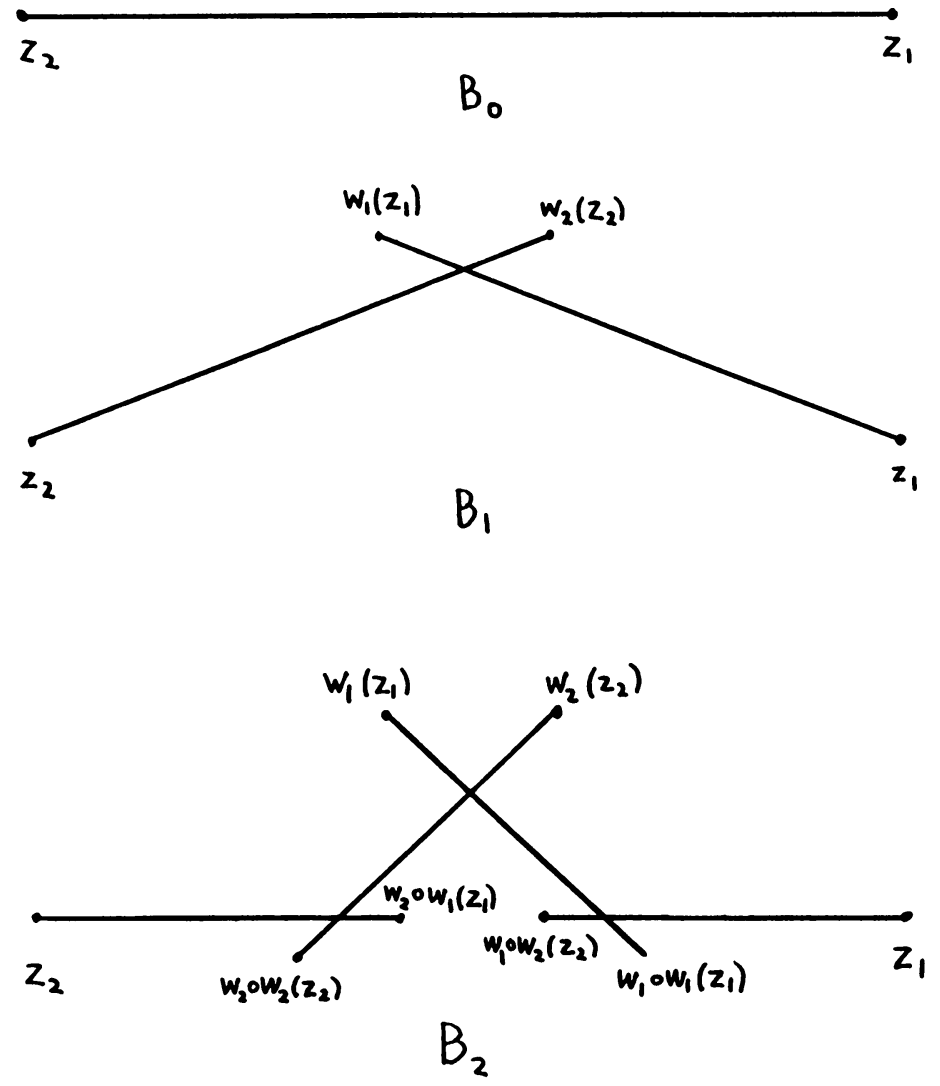

FIGURE 8

the angle $/ w_{1}\left(z_{1}\right) z_{1} w_{1} \circ w_{2}\left(z_{2}\right)$ measured from $\overline{z_{1} w_{1}\left(z_{1}\right)}$ in a ccw direction is $\pi-\theta(s)$. Thus $w_{1} \circ w_{2}\left(z_{2}\right)$ lies on $\overline{z_{1} z_{2}}$. Similarly, $/ w_{1} w_{1}\left(z_{1}\right) w_{1}\left(z_{1}\right) z_{1}$ measured from $\overline{z_{1} w_{1}\left(z_{1}\right)}$ in a ccw direction is $\pi-\theta(s)$. Let $P$ be a point on the line through $w_{1}\left(z_{1}\right)$ and $w_{2}\left(z_{2}\right)$ with $\operatorname{Re}[P]>\operatorname{Re}\left[w_{1}\left(z_{1}\right)\right] ; \operatorname{then} / P w_{1}\left(z_{1}\right) z_{1}$ measured from $\frac{1}{P w_{1}\left(z_{1}\right)}$ in a ccw direction is $\pi-\theta(s)$. Thus $/ P w_{1}\left(z_{1}\right) w_{1} w_{1}\left(z_{1}\right)$ is $2(\pi-\theta(s))$, which is between 0 and $\pi / 2$. Thus, $w_{1} \circ w_{1}\left(z_{1}\right) \in B$ and $\operatorname{Re}\left[w_{1}\left(z_{1}\right)\right] \leq \operatorname{Re}\left[w_{1} \circ w_{1}\left(z_{1}\right)\right] \leq \operatorname{Re}\left[w_{1} \circ w_{2}\left(z_{2}\right)\right] \leq \operatorname{Re}\left[z_{1}\right]$. Now $w_{1}(B)$ is the trapezoid with vertices $\left\{z_{1}, w_{1}\left(z_{1}\right), w_{1} \circ w_{1}\left(z_{1}\right), w_{1} \circ w_{2}\left(z_{2}\right)\right\}$, all of which we have shown to lie in $B$. Thus, $w_{1}(B) \subset B$. Furthermore, if $z \in w_{1}(B)$ then $\operatorname{Re}[z] \geq \operatorname{Re}\left[w_{1}\left(z_{1}\right)\right]>0$. Lemma 9 implies $w_{2}(B)=-\left(w_{1}(B)\right)^{*}$, so if $z \in w_{2}(B)$ then $\operatorname{Re}[z]<0$. Thus $w_{2}(B) \subset B$ and so $A(s)$ is disconnected.

The converse is also true.

Proposition 11. If $3 \pi / 4 \leq \theta(s) \leq \pi$ and $\operatorname{Re}\left[w_{1}\left(z_{1}\right)\right] \leq 0$ (equivalent $\operatorname{Re}[s] \leq$ $-.5)$ then $A(s)$ is connected. 


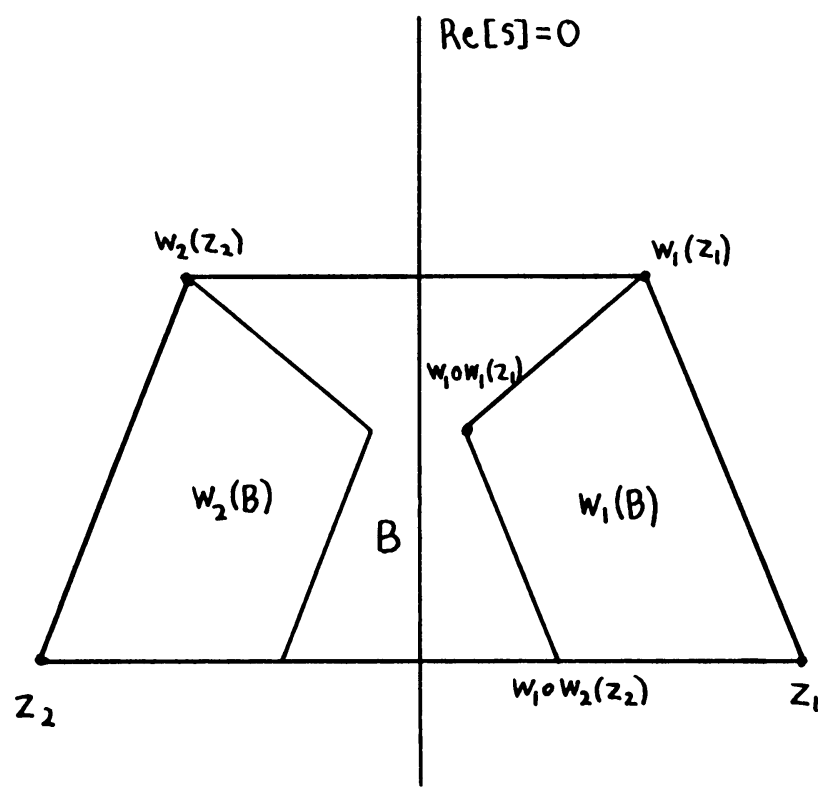

Figure 9

Proof. Let $B_{0}=\overline{z_{1} z_{2}}$ and $B_{n}=\underline{w}^{\circ n}\left(B_{0}\right)$ for $n \in \mathbf{N}$. Figure 8 shows $B_{0}, B_{1}$, $B_{2}$ for a typical $s$. We will first show that $B_{n}$ is connected.

Since $w_{2}\left(z_{1}\right)=z_{2}, w_{1}\left(z_{2}\right)=z_{1}$, and $z_{1}, z_{2} \in B_{0}$ then $z_{1}, z_{2} \in B_{n}$ and thus $w_{1}\left(z_{1}\right), z_{1} \in w_{1}\left(B_{n}\right)$ for $n \in \mathbf{N}$.

Note that $-B_{0}^{*}=B_{0}$. Suppose $-B_{n}^{*}=B_{n}$; then $-B_{n+1}^{*}=\left(-w_{1}\left(B_{n}\right)\right)^{*} \cup$ $\left(-w_{2}\left(B_{n}\right)\right)^{*}=w_{2}\left(-B_{n}^{*}\right) \cup w_{1}\left(-B_{n}^{*}\right)=\underline{w}\left(B_{n}\right)=B_{n+1}$. By induction $-B_{n}^{*}=B_{n}$ for $n \in \mathbf{N}$. Thus $-\left(w_{1}\left(B_{n}\right)\right)^{*}=w_{2}\left(-B_{n}^{*}\right)=w_{2}\left(B_{n}\right)$, so if $x \in w_{1}\left(B_{n}\right)$ and $\operatorname{Re}[x]=0$ then $x \in w_{2}\left(B_{n}\right)$.

Note that $B_{0}$ is connected. If $B_{n}$ is connected then $w_{1}\left(B_{n}\right)$ and $w_{2}\left(B_{n}\right)$ are connected. Recall that $w_{1}\left(z_{1}\right), z_{1} \in w_{1}\left(B_{n}\right)$, and that $\operatorname{Re}\left[z_{1}\right]>0$ and by hypothesis $\operatorname{Re}\left[w_{1}\left(z_{1}\right)\right] \leq 0$. By the intermediate value theorem there must be some $a \in w_{1}\left(B_{n}\right)$ with $\operatorname{Re}[a]=0$. But then $a \in w_{2}\left(B_{n}\right)$ so $w_{1}\left(B_{n}\right) \cap w_{2}\left(B_{n}\right) \neq$ $\varnothing$ and $\underline{w}\left(B_{n}\right)$ is connected. The proposition then follows from Lemma 2 .

Figures 9 and 10 illustrate the case for $\theta(s) \in[\pi / 2,3 \pi / 4]$. Now $w_{1} \circ w_{1}\left(z_{1}\right)$ plays the role that $w_{1}\left(z_{1}\right)$ played for $\theta(s) \in[3 \pi / 4, \pi]$.

Proposition 12. If $3 \pi / 4 \leq \theta(s) \leq \pi / 2$ then $A(s)$ is disconnected if and only if $\operatorname{Re}\left[w_{1} \circ w_{1}\left(z_{1}\right)\right]>0$.

Proof. Suppose $\operatorname{Re}\left[w_{1} \circ w_{1}\left(z_{1}\right)\right]>0$. Again let $B$ be the trapezoid with vertices $\left\{z_{1}, z_{2}, w_{1}\left(z_{1}\right), w_{2}\left(z_{2}\right)\right\}$. From the proof of Proposition 11 it still follows that $\underline{w}(B) \subset B$. Since $/ P w_{1}\left(z_{1}\right) w_{1} \circ w_{1}\left(z_{1}\right)=2(\pi-\theta(s)) \in[\pi / 2, \pi]$ we see that $\operatorname{Re}\left[w_{1} \circ w_{1}\left(z_{1}\right)\right] \leq \operatorname{Re}\left[w_{1}\left(z_{1}\right)\right]$. Since $/ w_{1} \circ w_{1}(z), w_{1} \circ w_{2}\left(z_{2}\right) z_{1}=\theta(s) \in$ $[3 \pi / 4, \pi / 2]$ we see that $\operatorname{Re}\left[w_{1} \circ w_{1}\left(z_{1}\right)\right] \leq \operatorname{Re}\left[w_{1} \circ w_{2}\left(z_{2}\right)\right] \leq \operatorname{Re}\left[z_{1}\right]$. Thus $0<\operatorname{Re}\left[w_{1} \circ w_{1}\left(z_{1}\right)\right] \leq \operatorname{Re}[z]$ for all $z \in w_{1}(B)$ and so $w_{1}(B) \cap w_{2}(B)=\varnothing$. 

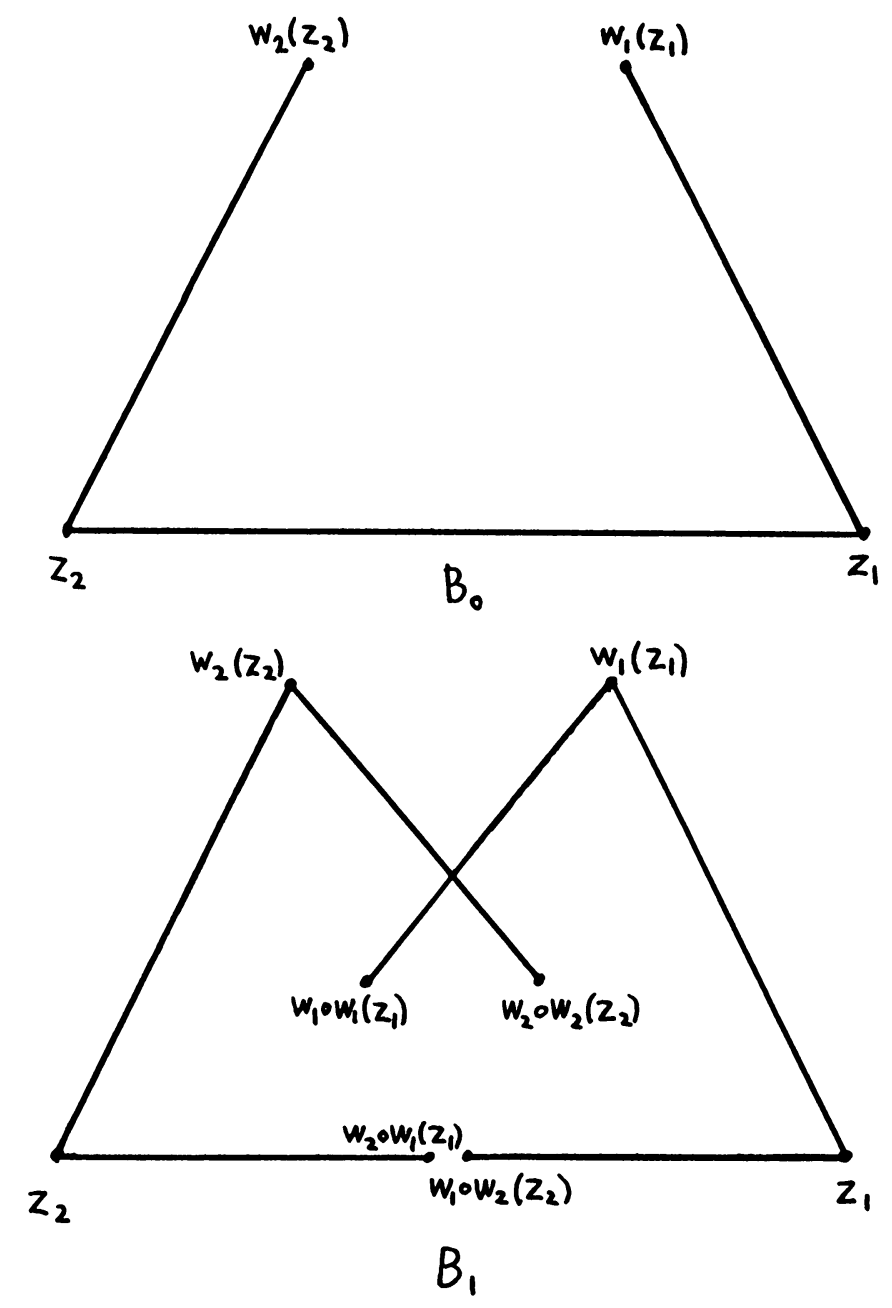

Figure 10

Now suppose $\operatorname{Re}\left[w_{1} \circ w_{1}\left(z_{1}\right)\right] \leq 0$. Let $B_{0}=\overline{w_{1}\left(z_{1}\right) z_{1}} \cup \overline{z_{1} z_{2}} \cup \overline{z_{2} w_{2}\left(z_{2}\right)}$ and let $B_{n}=\underline{w}^{o n}\left(B_{0}\right)$. By an induction $B_{n}$ is connected and so by Lemma 2 $A(s)$ is connected.

Proposition 13. If $n \in \mathbf{N}$ and $\pi /(2 n+2) \leq \theta(s) \leq \pi /(2 n)$ then $A(s)$ is disconnected if and only if $\operatorname{Re}\left[w_{1} \circ w_{2}^{o(n+1)}\left(z_{2}\right)\right]>0$.

Proof. Since the method of proof should be familiar by now, we will only outline the proof of this proposition. Figure 11 illustrates the case for $n=1$ and $n=2$.

Suppose $\operatorname{Re}\left[w_{1} \circ w_{2}^{\circ(n+1)}\left(z_{2}\right)\right]>0$. Let $B$ be the closed convex hull of $\left\{z_{1}, z_{2}, w_{1}\left(z_{1}\right), w_{2}\left(z_{2}\right), \ldots, w_{1}^{\circ(2 n+1)}\left(z_{1}\right), w_{2}^{\circ(2 n+1)}\left(z_{2}\right)\right\} ;$ then $\underline{w}(B) \subset B$ and if $z \in w_{1}(B)$ then $\operatorname{Re}(z) \geq \operatorname{Re}\left(w_{1} \circ w_{2}^{\circ(n+1)}\left(z_{2}\right)\right)>0$. Thus $w_{1}(B) \cap w_{2}(B)=\varnothing$ and so $A(s)$ is disconnected. 

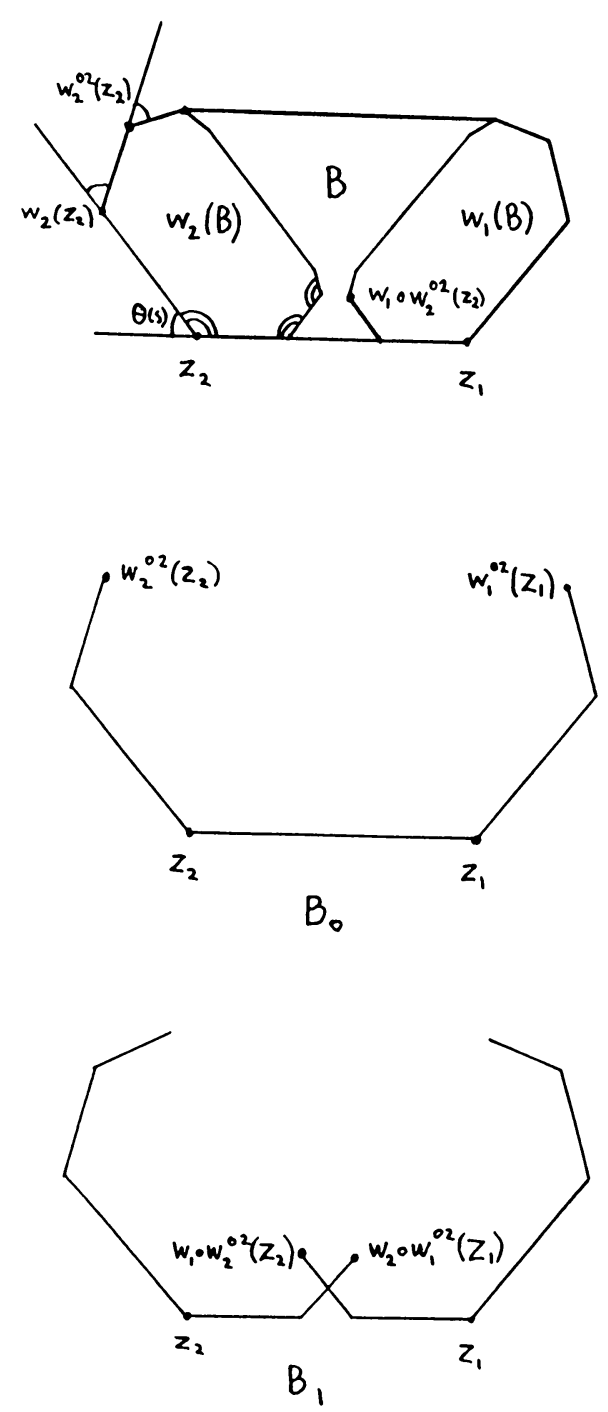

$\pi / 4 \leq \theta(s) \leq \pi / 2$
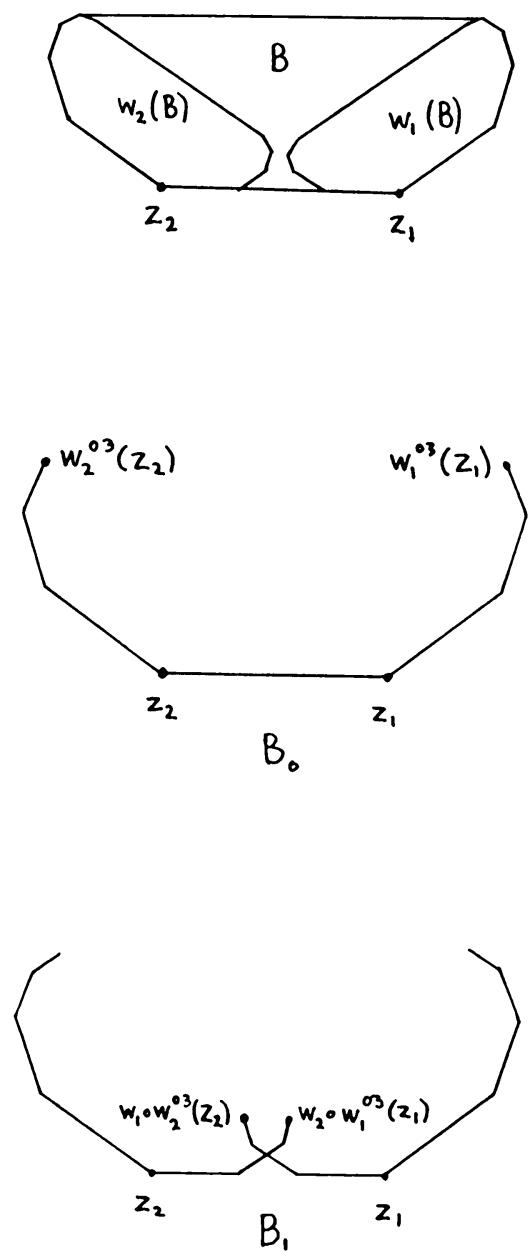

$\pi / 6 \leq \theta(s) \leq \pi / 4$

FIGURE 11

Suppose $\operatorname{Re}\left[w_{1} \circ w_{2}^{\circ(n+1)}\left(z_{2}\right)\right] \leq 0$. Let

$$
B_{0}=\overline{z_{1} z_{2}} \cup\left\{\bigcup_{i=0}^{n} \overline{\left(w_{1}^{\circ(i+1)}\left(z_{1}\right) w_{1}^{\circ(i)}\left(z_{1}\right)\right.} \cup \overline{\left.w_{2}^{\circ(i+1)}\left(z_{2}\right) w_{2}^{o(i)}\left(z_{2}\right)\right)}\right\}
$$

An induction shows that $\underline{w}^{\circ k}\left(B_{0}\right)$ is connected for all $k \in \mathbf{N}$, so $A(s)$ is connected.

The conditions given in Propositions 10-13 that $s$ be on $\partial G$ can be expressed as polynomial curves in $x=\operatorname{Re}[s]$ and $y=\operatorname{Im}[s]$. For $|s|<1$, 
$\operatorname{Re}\left[w_{1}\left(z_{1}\right)\right]=0$ if and only if $x=-.5$, and $\operatorname{Re}\left[w_{1} \circ w_{1}\left(z_{1}\right)\right]=0$ if and only if $2 x+2 x+1-2 y^{2}=0$. Note that $\operatorname{Re}\left[w_{1} \circ w_{2}^{\circ(n+1)}\left(z_{2}\right)\right]=0$ if and only if

$$
\operatorname{Re}\left[|s|^{2}\left(s^{n}-s^{n+1}\right)+\left(1-|s|^{2}\right)\left(|s|^{2} \sum_{p=0}^{n} s^{p-1}-1\right)\right]=0,
$$

which describes a polynomial curve for each $n \in \mathbf{N}$. We will now use these conditions to prove our main result.

Theorem 14. $G$ is connected.

Proof. We will show that for each $\theta(s) \in[0, \pi]$ there is an $r^{*} \in(0,1)$ such that $s \in G$ if and only if $|s|<r^{*}$. Recall that we already know that $s \in G$ if $|s|<.5$ and that $s \notin G$ if $|s|>1 / \sqrt{2}$. Thus we need only show that the appropriate function (for instance $\operatorname{Re}\left[w_{1} \circ w_{1}\left(z_{1}\right)\right]$ for $\theta(s) \in[\pi / 2,3 \pi / 4]$ ) can be zero at most once in the interval $(.5,1 / \sqrt{2}]$.

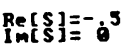

$\operatorname{Re}(s)=-15$

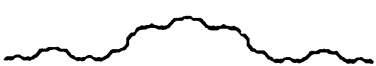

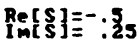

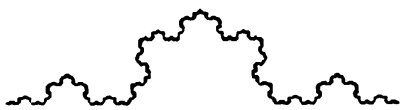

\section{Rets 15$]=-: 575$}

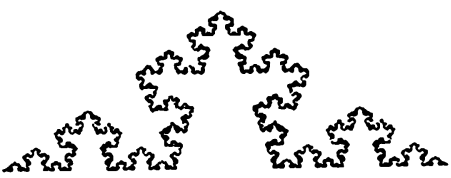

\section{$\operatorname{Re}[\{]=-:\{$}

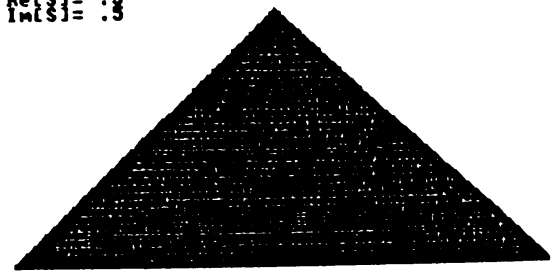

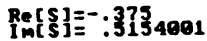

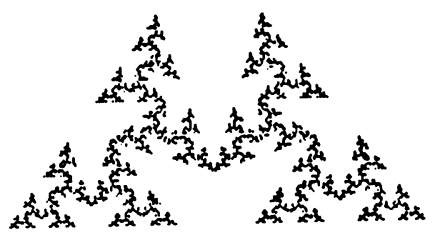

Figure 12. $A(s)$ as $s$ varies along $\partial G$ 


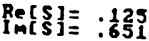

$\operatorname{Re}[s]=-: 35$,
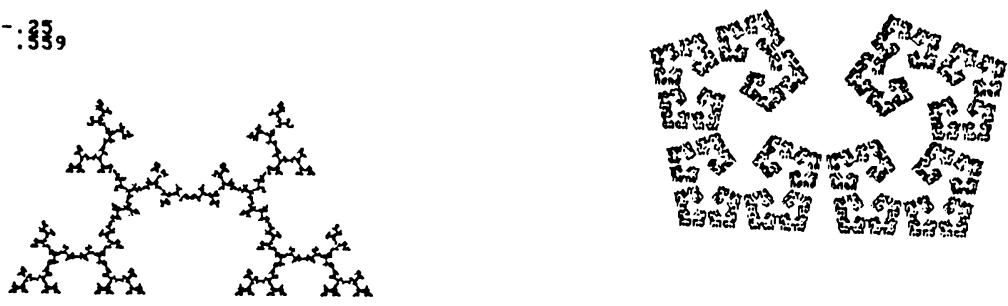

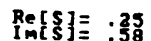

$\operatorname{Rec}(\$ 1)=-125$
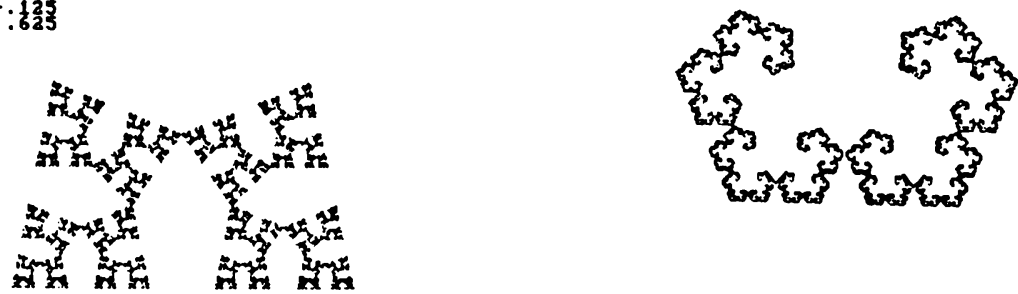

$\operatorname{Re}\left[\begin{array}{l}\text { Re } \\ \ln [s] \equiv: 375\end{array}\right.$

$\operatorname{Re}[s] \equiv:$ : 2070999
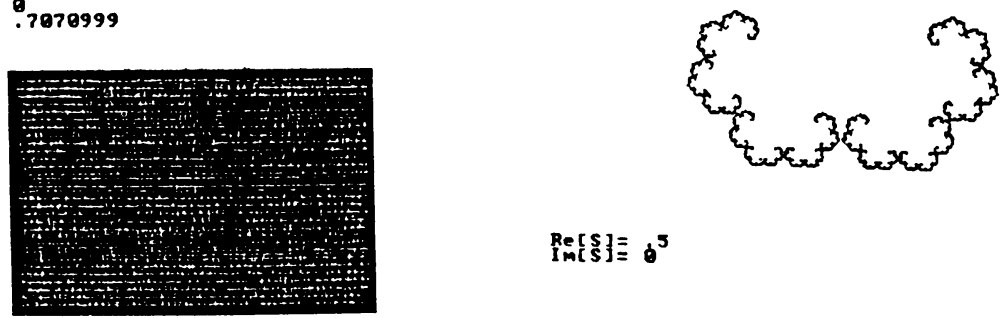

$\operatorname{Re}\left[\begin{array}{l}\text { Re } \\ \ln \end{array}\right] \equiv \dot{\theta}^{5}$

FIGURE 12 (continued)

Case 1. $\theta(s) \in[3 \pi / 4, \pi]$ : Propositions 10 and 11 imply $s \in G$ if and only if $\operatorname{Re}[s]<.5 ;$ however, $\operatorname{Re}[s]<.5$ if and only if $|s|<.5|\sec \theta(s)| \equiv r^{*}$.

Case 2. $\theta(s) \in[\pi / 2,3 \pi / 4]:$ By Proposition 12, $s \in G$ if and only if $\operatorname{Re}\left[w_{1} \circ w_{1}\left(z_{1}\right)\right]=0$. Define

$f(r)=\operatorname{Re}\left[w_{1} \circ w_{1}\left(z_{1}\right)\right]=\left[r^{2} /\left(1-r^{2}\right)\right][\cos 2 \theta(s)-r \cos 3 \theta(s)]+r \cos \theta(s)+1$,

where $r=|s|$. Since $\cos \theta(s)<0, \cos 2 \theta(s)<0$, and $\cos 3 \theta(s)>0$, it is clear that $f(r)$ is a decreasing function for $r \in(0,1)$ and thus can be zero at most once in the interval $(.5,1 / \sqrt{2}]$. 
Case 3. $\theta(s) \in(0, \pi / 2]$ : Let $n$ be such that $\pi / 2^{n+1} \leq \theta(s) \leq \pi / 2^{n}$. Now $s \in G$ if and only if $\operatorname{Re}\left[w_{1} \circ w_{2}^{\circ(n+1)}\left(z_{2}\right)\right]>0$. Define

$$
\begin{aligned}
f(r)= & \operatorname{Re}\left[w_{1} \circ w_{2}^{\circ(n+1)}\left(z_{2}\right)\right] \\
= & \left(r^{(n+3)} \cos [(n+1) \theta(s)]-r^{(n+2)} \cos [n \theta(s)]\right) /\left(1-r^{2}\right) \\
& -\left(\sum_{\dot{p}=0}^{n} r^{p+1} \cos [(p-1) \theta(s)]\right)+1 .
\end{aligned}
$$

It is a short exercise in freshman calculus to show that $f(r)$ is decreasing on $(.5,1 / \sqrt{2})$.

Case 4. $\theta(s)=0: A(s)$ is an interval if $|s| \geq .5$ and a Cantor set if $|s|<.5$, so $s \in G$ if and only if $|s|<.5$.

Since $G$ is symmetric about the real axis, we see that $G$ is connected.

$A$ tour around the boundary of $G$. The evolution of $A(s)$ as $s$ varies along $\partial G$ is rather interesting. Figure 12 shows $A(s)$ at various values of $s$ on $\partial G$. Note that $A(s)$ consists of a family of Koch [8] curves as $s$ varies from -.5 to $-.5+i .5$, at which point $A(s)$ is a right triangle. The other interesting point is $s=i / \sqrt{2}$, where $A(s)$ is a rectangle.

The family of attractors for $\theta(s) \in(0, \pi / 2)$ includes fractals which arise as natural boundaries in the complex $t$-plane for nonintegrable dynamical systems $[9,10]$. In fact, these fractals provided our original motivation for studying this particular family.

\section{APPENDIX}

In this appendix we present a computer program which generates computer images of $D$. The program can be used with minor modifications to find the Mandelbrot set for any family of pairs of similitudes on $\mathbf{R}^{2}$.

The program runs on the IBM PC microcomputer in compiled BASIC. A typical picture is produced in approximately 12 hours when the number of iterations is between 10 and 15. The program is much slower in regions which are near $\partial D$ and which are near the real axis. 
10 DIM AX (4), AY(4), X(2,2,30), Y(2,2,30),P(2,30), RSC(30), LN(30)

20 INPUT "window in parameter space $\mathrm{a}<\operatorname{Re}[\mathrm{S}]<\mathrm{b} ; \mathrm{c}<\operatorname{Im}[\mathrm{S}]<\mathrm{d}^{n} ; \mathrm{AA}, \mathrm{BB}, \mathrm{CC}, \mathrm{DD}$

30 INPUT "pixel window; px1,px2,py 1,py 2 where $0<=\mathrm{px} 1<\mathrm{px} 2<320$ and $0<=$ py $1<$ py $2<200$ (e.g. 40,279,0,199 gives a square) ${ }^{n}$;PX1,PX2,PY1,PY2

40 INPUT "file name for picutre";PICFILE $\$$

50 INPUT "number of iterations $<=30^{n} ; \mathrm{NUMIT}$

$60 \mathrm{HX}=(\mathrm{BB}-\mathrm{AA}) /(\mathrm{PX} 2-\mathrm{PX} 1): \mathrm{HY}=(\mathrm{DD}-\mathrm{CC}) /(\mathrm{PY} 2-\mathrm{PY} 1)$

70 SCREEN 1,0:KEY OFF: CLS

$80 \mathrm{P}(1,0)=1: \mathrm{P}(2,0)=2$

90 FOR SY $=$ CC TO DD STEP HY

100 FOR SX=AA TO BB STEP HX

$110 \mathrm{PSX}=(\mathrm{PX} 2-\mathrm{PX} 1)^{*}(\mathrm{SX}-\mathrm{AA}) /(\mathrm{BB}-\mathrm{AA})+\mathrm{PX} 1: \mathrm{PSY}=(\mathrm{PY} 2-\mathrm{PY} 1)^{*}(\mathrm{DD}-\mathrm{SY}) /(\mathrm{DD}-\mathrm{CC})+\mathrm{PY} 1$

$120 \mathrm{SC}=\mathrm{SX} * \mathrm{SX}+\mathrm{SY}{ }^{*} \mathrm{SY}$

130 IF SC $>.5$ THEN GOTO 300

$140 \mathrm{RSC}(0)=\mathrm{SC} /(1-\mathrm{SQR}(\mathrm{SC}))^{2}$

150 FOR $\mathrm{K}=1$ TO NUMIT

$160 \mathrm{RSC}(\mathrm{K})=\mathrm{RSC}(\mathrm{K}-1) * \mathrm{SC}$

170 NEXT K

180 IF RSC $(0)<1$ THEN COLCODE=3:GOTO 370

$190 \mathrm{~N}=1: \mathrm{COLCODE}=1$

$200 \mathrm{LN}(\mathrm{N})=1: \mathrm{WP}=1: \mathrm{P}(1, \mathrm{~N})=1:$ GOSUB 380

210 IF COLCODE $<N$ THEN COLCODE $=N$

$220 \mathrm{P}(2, \mathrm{~N})=1: \mathrm{WP}=2: \mathrm{GOSUB} 380$

$230 \mathrm{~A}=1$ : $\mathrm{B}=1$ :GOSUB 520: IF DST $<=\mathrm{RSC}(\mathrm{N})$ THEN GOTO 290

$240 \mathrm{LN}(\mathrm{N})=2: \mathrm{WP}=2: \mathrm{P}(2, \mathrm{~N})=2:$ GOSUB 380

$250 \mathrm{~A}=1 \mathrm{~B}=2$ :GOSUB 520: IF DST $<=\mathrm{RSC}(\mathrm{N})$ THEN GOTO 290

$260 \mathrm{LN}(\mathrm{N})=3: \mathrm{WP}=1: \mathrm{P}(1, \mathrm{~N})=2:$ GOSUB 380

$270 \mathrm{~A}=2: \mathrm{B}=1: \mathrm{P}(2, \mathrm{~N})=1:$ GOSUB $520: \operatorname{IF}$ DST $<=\mathrm{RSC}(\mathrm{N})$ THEN GOTO 290

$280 \mathrm{LN}(\mathrm{N})=4: \mathrm{A}=2: \mathrm{B}=2: \mathrm{P}(2, \mathrm{~N})=2$ :GOSUB 520:IF DST $>$ RSC $(\mathrm{N})$ THEN GOTO 550

290 IF $\mathrm{N}<\mathrm{NUMIT}$ THEN $\mathrm{N}=\mathrm{N}+1:$ GOTO 200

300 NEXT SX

310 NEXT SY

320 DEF SEG $=\& H B 800$ :BSAVE PICFILE $\$, 0, \& H 4000$

330 INPUT WONT

340 IF WONT THEN GOTO 20

350 END

$360 \mathrm{COLCODE}=\mathrm{COLCODE} \mathrm{MOD} 3+1$

370 PSET (PSX,PSY),COLCODE : GOTO 300

$380 \mathrm{XX}=0: \mathrm{YY}=0$

390 FOR $\mathrm{K}=0$ TO N

400 ON P(WP,N-K) GOSUB 440,480

410 NEXT K

$420 X(W P, P(W P, N), N)=X X: Y(W P, P(W P, N), N)=Y Y$

430 RETURN

$440 \mathrm{XN}=\mathrm{SX}^{*} \mathrm{XX}-\mathrm{SY} \mathrm{Y}^{*} \mathrm{Y}+1$

$450 \mathrm{YY}=\mathrm{SX} \mathrm{X}^{*} \mathrm{YY}+\mathrm{SY}^{*} \mathrm{XX}$

$460 \mathrm{XX}=\mathrm{XN}$

470 RETURN

$480 \mathrm{XN}=\mathrm{SX} \mathrm{XXX}^{*} \mathrm{SY} \mathrm{Y}^{*} \mathrm{Y}-1$

$490 \mathrm{YY}=\mathrm{SX} \mathrm{X}^{*} \mathrm{YY}+\mathrm{SY}^{*} \mathrm{XX}$

$500 \mathrm{XX}=\mathrm{XN}$

510 RETURN

$520 \mathrm{DELX}=\mathrm{X}(1, \mathrm{~A}, \mathrm{~N})-\mathrm{X}(2, \mathrm{~B}, \mathrm{~N}): \mathrm{DELY}=\mathrm{Y}(1, \mathrm{~A}, \mathrm{~N})-\mathrm{Y}(2, \mathrm{~B}, \mathrm{~N})$

$530 \mathrm{DST}=.25^{*}\left(\mathrm{DELX}{ }^{*} \mathrm{DELX}+\mathrm{DELY}{ }^{*} \mathrm{DELY}\right)$

540 RETURN

550 IF $N=1$ THEN GOTO 360

$560 \mathrm{~N}=\mathrm{N}-1:$ ON LN(N) GOTO $240,260,280,550$ 


\section{REFERENCES}

1. B. Mandelbrot, Fractal aspects of the iteration of $z \rightarrow \lambda z \cdot(1-z)$, Ann. New York Acad. Sci. 357 (1980), 249-259; On the quadratic mapping $z \rightarrow z^{2}-\mu$ for complex $\mu$ and $z:$ the fractal structure of its $M$ set and scaling, Physica 7D (1983), 224-239; On the dynamics of iterated maps VIII, Chaos and Statistical Methods (Y. Kuramoto, ed.), Springer, Berlin, 1984, pp. 32-42.

2. A. Douady and J. Hubbard, C. R. Acad. Sci. Paris 294 (1982), 123-126.

3. A. Douady, Systèmes dynamique holomorphes, Sem. Bourbaki 35 (599) (1982/1983).

4. M. Feigenbaum, Quantitative universality for a class of nonlinear transformations, J. Statist. Phys. 19 (1978), 25-52; P. J. Myrberg, Sur l'iteration des polynomes réels quadratiques, J. Math. Pures Appl. (9) 41 (1962), 339-351.

5. M. F. Barnsley and A. N. Harrington, Physica 15D (1985), 421-432.

6. J. Hutchinson, Indiana Univ. Math. J. 30 (1981), 713-747.

7. M F. Barnsley and S. G. Demko, Iterated function systems and global construction of fractals, Proc. Roy. Soc. London Ser. A 399 (1985), 243-275.

8. B. Mandelbrot, The fractal geometry of nature, Freeman, San Francisco, Calif., 1983.

9. H. Yoshida, Self-similar natural boundaries of nonintegrable dynamical systems in the complex t-plane, Preprint, Dept. of Astronomy, Univ. of Tokyo.

10. D. Bessis and N. Chafee, On the existence and non-existence of natural boundaries for nonintregrable dynamical systems, Chaotic Dynamics and Fractals (M. F. Barnsley and S. G. Demko, eds.), Academic Press, New York, 1985.

School of Mathematics, Georgia Institute of Technology, Atlanta, Georgia 30332 (Current address of M. F. Barnsley)

Current address (D. P. Hardin): Department of Mathematics, Vanderbilt University, Nashville, Tennessee 37235 